\title{
REVIEW ARTICLE \\ Subclinical infection and asymptomatic carriage of gastrointestinal zoonoses: occupational exposure, environmental pathways, and the anonymous spread of disease
}

\author{
R. S. QUILLIAM ${ }^{1 *}$, P. CROSS ${ }^{2}$, A. PRYSOR WILLIAMS ${ }^{2}$, \\ G. EDWARDS-JONES ${ }^{2} \dagger$, R. L. SALMON ${ }^{3}$, D. RIGBY ${ }^{4}$, R. M. CHALMERS ${ }^{5}$, \\ D. RH. THOMAS ${ }^{3}$ AND D. L. JONES ${ }^{2}$ \\ ${ }^{1}$ Biological and Environmental Sciences, School of Natural Sciences, University of Stirling, Stirling, UK \\ ${ }^{2}$ School of Environment, Natural Resources and Geography, College of Natural Sciences, Bangor University, \\ Bangor, Gwynedd, UK \\ ${ }^{3}$ Communicable Disease Surveillance Centre, Public Health Wales, Temple of Peace and Health, Cathays Park, \\ Cardiff, $U K$ \\ ${ }^{4}$ School of Social Sciences, University of Manchester, Manchester, UK \\ ${ }^{5}$ UK Cryptosporidium Reference Unit, National Public Health Service Microbiology Swansea, Singleton \\ Hospital, Swansea, UK
}

Received 28 September 2012; Final revision 19 December 2012; Accepted 17 April 2013;

first published online 10 May 2013

\section{SUMMARY}

Asymptomatic carriage of gastrointestinal zoonoses is more common in people whose profession involves them working directly with domesticated animals. Subclinical infections (defined as an infection in which symptoms are either asymptomatic or sufficiently mild to escape diagnosis) are important within a community as unknowing (asymptomatic) carriers of pathogens do not change their behaviour to prevent the spread of disease; therefore the public health significance of asymptomatic human excretion of zoonoses should not be underestimated. However, optimal strategies for managing diseases where asymptomatic carriage instigates further infection remain unresolved, and the impact on disease management is unclear. In this review we consider the environmental pathways associated with prolonged antigenic exposure and critically assess the significance of asymptomatic carriage in disease outbreaks. Although screening high-risk groups for occupationally acquired diseases would be logistically problematical, there may be an economic case for identifying and treating asymptomatic carriage if the costs of screening and treatment are less than the costs of identifying and treating those individuals infected by asymptomatic hosts.

Key words: Acquired immunity, community epidemiology, disease management, environmental medicine, public health.

\section{INTRODUCTION}

Each year, foodborne diseases account for an estimated 1.3 million cases in England and Wales and

\footnotetext{
* Author for correspondence: Dr R. S. Quilliam, Biological and Environmental Sciences, School of Natural Sciences, University of Stirling, Stirling, FK9 4LA, UK.

(Email: Richard.Quilliam@stir.ac.uk)

$\dagger$ Deceased.
}

76 million cases in the USA [1, 2], with four of the most important pathogens responsible for foodborne illnesses being zoonotic (Campylobacter spp., Salmonella spp., Cryptosporidium spp. and verocytotoxin-producing Escherichia coli (VTEC), particularly E. coli O157). In addition to causing acute diarrhoeal symptoms, infection may lead to more severe complications, e.g. haemolytic uraemic syndrome from E. coli O157, and Guillain-Barré 
Table 1. Occupational and environmental risk exposures associated with gastrointestinal zoonotic pathogens

\begin{tabular}{lll}
\hline \hline Zoonotic disease & Occupational and environmental risk exposures & References \\
\hline Campylobacteriosis & $\begin{array}{l}\text { Poultry abattoir workers } \\
\text { Living or working on a farm (particularly poultry) } \\
\text { Recreational water activities }\end{array}$ & {$[11,14-16,18-20]$} \\
Cryptosporidiosis & $\begin{array}{l}\text { Visiting farms and contact with farm animals } \\
\text { Recreational water activity (particularly swimming pools) }\end{array}$ & {$[25-28,31]$} \\
& $\begin{array}{l}\text { Living or working on a farm (dairy) } \\
\text { Rural areas with high ruminant livestock density }\end{array}$ & \\
Salmonellosis & Living or working on a farm & \\
& $\begin{array}{l}\text { Farm contact (sheep and dairy) } \\
\text { Working in a veterinary clinic }\end{array}$ & \\
VTEC (including O157) & Living or working on a farm (sheep and dairy) \\
& Rural residency or farm contact (particularly with animal faeces) \\
& Visiting agricultural fairs and petting zoos & \\
& Meat processors and abattoir workers & \\
\hline \hline
\end{tabular}

VTEC, Verocytotoxin-producing Escherichia coli.

syndrome from Campylobacter infection. Although many cases of human salmonellosis, campylobacteriosis, cryptosporidiosis and E. coli $\mathrm{O} 157$ infections are foodborne or waterborne, both outbreak and sporadic infections have been linked with the veterinary surgeries and the farming environment and a number of associated risk exposures have been identified (Table 1). Here we consider the environmental pathways associated with prolonged antigenic exposure and assess the role of occupational exposure in susceptibility to zoonotic pathogens. We discuss the significance of asymptomatic carriage in disease outbreaks, and explore the epidemiological implications of asymptomatic carriage on disease management.

Zoonoses are infectious diseases that are transmissible from vertebrate animals to humans. In many countries, the reporting of most zoonotic illness has traditionally been voluntary and therefore laboratoryconfirmed zoonotic cases probably account for only a small fraction of those occurring in the community. Farm workers, veterinarians and abattoir workers, who have more repeated contact with animals than almost any other occupational group, have a higher potential risk of contracting zoonotic illnesses. Seroprevalence studies have shown that these groups and their families are at risk of exposure to a significant number of zoonoses [3-5]. However, frequent and recurrent occupational exposure to zoonoses can result in reduced occurrence of clinical illness due to a build-up of immunity; consequently it has been suggested that asymptomatic carriage of zoonoses is more likely in people whose occupation involves them working directly with domesticated or wild animals.

Despite the lack of clinical symptoms, individuals with apparent immunity can still be colonized by zoonotic pathogens [6], and could inadvertently facilitate disease transmission. A recent study from India demonstrated that children excreting viruses asymptomatically served as a significant source of infection because they were not restricted by illness [7]. The public health significance of this should not be underestimated as asymptomatically colonized individuals involved in the food supply chain will not necessarily alter their behaviour (e.g. due to diarrhoea) and could be responsible for the prolonged cycling and transmission of infectious diseases. The costs of illness to local health services and the wider economy associated with asymptomatic carriage are currently unknown. A better understanding of the scale of the role of asymptomatic carriage in disease burden would inform policy makers and allow a more accurate assessment of the value and cost-effectiveness of intervention strategies which would address it.

\section{Recurrent occupational animal exposure and subclinical infection}

E. coli $\mathrm{O} 157$

Circumstantial evidence suggests that occupational exposure to domesticated livestock can result in subclinical infection with VTEC. For example, a study in Canada found that $12 \%$ of dairy farm families 
possessed serum antibodies to the E. coli O157 lipopolysaccharide (LPS), although none of these subjects reported gastrointestinal symptoms at the time of sampling or a history of bloody diarrhoea or renal problems [8]. Further evidence of asymptomatic carriage of VTEC in farm workers has led to the suggestion that a degree of immunity to clinical infection by E. coli $\mathrm{O} 157$ develops as a result of prolonged lowlevel exposure to less virulent VTEC strains [6]. This was supported by a retrospective serosurvey of healthy adult farm workers from the UK, which provided evidence of persisting antibodies to E. coli O157, particularly of the IgG class, suggesting long-term or repeated exposure to VTEC [9]. Further studies have shown elevated levels of antibodies to E. coli $\mathrm{O} 157$ LPS in dairy farm residents compared to urban residents [10], and in farm-resident rural children compared to non-farm-resident children [11]. Although the phenomenon of seropositivity and asymptomatic carriage of VTEC in farm workers has been well documented, the risk factors associated with carriage are still poorly understood $[12,13]$.

\section{Campylobacter}

While increased anti-Campylobacter jejuni antibodies are often detected in the sera of individuals who work with poultry $[14,15]$, the risk of developing Campylobacter infection is much greater in inexperienced or temporary poultry farm workers $[16,17]$. In a serological study of poultry abattoir workers, long-term occupational exposure was accompanied by high levels of $C$. jejuni-specific $\operatorname{IgG}$ antibodies [18]. Eight of these abattoir workers tested positive for faecal excretion of C. jejuni, with seven of them apparently asymptomatic, despite evidence for persistent excretion for up to 6 weeks. Occupational exposure to farm animals (e.g. cattle, sheep, poultry) and farm residency have also been identified as significant risk factors for Campylobacter infection [19-21]. However, it has also been suggested that occupational contact with livestock or their faeces can provide protection against Campylobacter disease [22]. This is supported by a study in rural Wisconsin, which showed that $59 \%$ of children possessed antibodies to $C$. jejuni, with farm-residency and increasing age independently associated with seropositivity, despite no corresponding increase in clinically recognized diarrhoeal illness compared to non-farm-resident children [11]. Other factors associated with seropositivity included contact with cattle and sheep and drinking unpasteurized milk, which in previous studies has also been associated with elevated levels of antibodies and immunity to Campylobacter symptomatic infection [23, 24]. However, while frequent farm-related antigenic stimulation may offer a degree of protective immunity, this type of acquired immunity is often incomplete, dose-respondent, and can be overcome by exposure to novel antigenic serotypes.

\section{Cryptosporidium}

Infection with the protozoan parasite Cryptosporidium $(\mathrm{Cr}$.) can cause gastrointestinal disease of clinical and economic significance in both humans and young farmed animals, with most human cases being caused by either $C r$. hominis, or Cr. parvum. Several UK studies have provided evidence for the anthroponotic spread of Cr. hominis and the mainly zoonotic spread of Cr. parvum, with short visits to farms, handling cattle and touching farm animals being identified as independent risk factors [25-27]. Furthermore, a quarter of all reported sporadic annual cases of Cr. parvum in England and Wales can be attributed to direct contact with farm animals [28]. Several seroepidemiological studies have found more positive subjects in rural settings than urban groups [e.g. 29, 30] and increased seroprevalence has been detected in dairy farmers compared to control subjects [31]. However, in a series of 790 sporadic cases in the UK between 2004 and 2006, only three cases were adults who had occupational farm contact (two farmers and one veterinary student), suggesting potential immunity in those frequently and historically exposed to animals and their faeces [28]. This contrasts with cryptosporidiosis outbreaks that regularly occur at swimming pools, daycare nurseries, open farms and similar settings which frequently involve children, and have been reported in veterinary students who have had limited prior exposure [32, 33]. Frequent exposure through intermittent low-level contamination may lead to subclinical infection and some herd immunity [34]. This is supported by evidence from a seroepidemiological study, which showed that people with high anti-cryptosporidial antibody levels were much less likely to self-report diarrhoeal disease than those with low levels of antibody [35]. Human infectivity studies have shown that although prior exposure does not provide complete protection against further infection, clinical symptoms are less severe $[36,37]$ and age-related increases in seroprevalence appear to 
correlate well with the decline in incidence of clinical cryptosporidiosis [37].

\section{Salmonella}

The routes of zoonotic transfer of Salmonella spp. include direct contact with farm animals or their faeces and exposure to a number of companion animals, particularly reptiles. Consequently, occupational exposure has been demonstrated in farm workers [38, 39] and veterinarians [40, 41], although the type of practice, i.e. small or large animal practice, will determine the Salmonella serotypes personnel are exposed to. Although secondary transmission of Salmonella is a recurrent problem in animals at veterinary clinics, there have been no direct studies on the levels of immunity in veterinary personnel; however, a stool sample from an asymptomatic employee during one outbreak of salmonellosis at a veterinary clinic in the USA was found to be positive for $S$. Typhimurium [41]. Clearly the risks for zoonotic transfer are high in veterinary workers, although a recent report indicated that veterinarians rarely use personal protective equipment or adopt infection control practices to protect themselves against zoonotic disease transmission [42]. It remains unclear whether the occupational exposure to zoonoses experienced by veterinary workers results in a form of protection; and although several studies have linked the zoonotic spread of Salmonella from farm animals to farm workers, intriguingly there are as yet no reports of asymptomatic carriage in farm workers. Whether this is due to a lack of cumulative exposure, through common antigens from the range of different Salmonella serotypes present in animals (as suggested for acquired immunity to E. coli $\mathrm{O} 157$ [6]), or whether this is simply because there is a paucity of studies clearly needs further epidemiological investigation.

\section{Acquired immunity and environmental exposure}

Exposure to potentially 'unhygienic' environments and the onset of protective immunity has been discussed in the context of many diseases. For example, evidence of acquired, exposure-related, immunity comes from travellers from Western countries who often suffer from 'traveller's diarrhoea' on arrival in a developing country [43-45]; however, their duration of stay is linearly correlated with protection from infection, indicating a build-up of immunity over time [46]. This supports a widely reported phenomenon in the abattoir industry where new employees with a lack of previous exposure to a range of zoonotic pathogens, almost inevitably report gastrointestinal illness in the first few days of employment, with far fewer cases occurring in longer-term employees [18, 47]. Although the implications of abattoir workers asymptomatically carrying gastrointestinal zoonotic pathogens is not as immediately serious as subclinical infection in other types of food handlers, e.g. during the preparation of ready-to-eat products, asymptomatic individuals may well be significantly facilitating the transmission of zoonotic pathogens into the food chain.

While few studies directly compare the prevalence of zoonoses within populations of developing and developed countries, there is evidence for increased asymptomatic carriage of gastrointestinal pathogens in developing nations [48, 49]. Environmental pathways associated with prolonged antigenic exposure include the recurring consumption of contaminated drinking water and lower levels of hygiene during food preparation together with an increased environmental burden. This makes recurring cycles of infection with a range of zoonoses in the developing world more likely, with many reports ascribing the asymptomatic carriage of particular zoonotic pathogens (e.g. Campylobacter) to this constant antigenic exposure [45]. Although microbial stimulation plays an important role in modulating and improving the immune response against future challenges by infective agents, the levels of immunity to zoonoses in the developing world are acquired at the expense of considerable infant and child mortality, often due to diarrhoeal diseases.

Asymptomatic Campylobacter infections are considered endemic in developing countries, with symptomatic infection limited to children aged $<2$ years. A considerable number of studies have reported that breastfeeding can induce basal immunity against certain zoonotic pathogens, e.g. Campylobacter [50], and as breastfeeding is widespread in the developing world, this may play an important role in the level of subclinical infection in children and asymptomatic carriage within a community. Conversely, evidence suggests that asymptomatic breastfeeding mothers can transfer certain zoonoses to their baby either directly, e.g. brucellosis [51, 52] or indirectly, e.g. Helicobacter pylori [53].

A large Iranian study conducted on faecal samples from children revealed that a significant proportion $(7 \cdot 2 \%)$ were asymptomatically shedding strains of 
enterotoxigenic E. coli (ETEC) known to cause diarrhoea [54]. A systematic review on the aetiological role of ETEC in children with diarrhoea in the developing world found that colonization in the $0-4$ years age group was a significant risk factor for contracting diarrhoea [44]. There also existed high rates of ETEC in children aged $>5$ years; however, these children were asymptomatic with a notable decrease in the frequency of diarrhoea. Enterohaemorrhagic E. coli O157, which has recently become a serious zoonotic pathogen in industrialized nations, appears to be a relatively uncommon causative agent of illness in developing countries. Whether this is due to this particular strain of $E$. coli being relatively new, or whether people in developing countries have already acquired a significant amount of immunity to it clearly warrants further investigation.

Genetic variation in human populations also plays an important role in susceptibility to infectious diseases, with several genetically determined factors influencing susceptibility to specific infections, e.g. Norwalk virus [55]. However, the role of genetic variation in determining resistance to infection by zoonotic pathogens remains unclear, although evidence from genetic epidemiological studies has demonstrated that immunity to disease usually arises from a complex interaction of environmental, pathogen and host genetic factors [56]. Genetically controlled host defence factors can affect the susceptibility of individuals to zoonotic challenge, e.g. individuals unable to produce or respond to interferon-gamma [57]. Genetic factors can also alter innate immunity, e.g. the heterogeneous condition hypogammaglobulinaemia can lead to repeated infection and prolonged symptoms from a range of pathogens, including zoonoses [58].

Comparing the incidence of zoonoses in immigrant and resident populations may help to understand acquired immunity, although these studies need to be treated with caution as they can often be influenced by confounding factors. An example of this occurred during the largest ever outbreak of $\mathrm{Q}$ fever in the UK, where no cases were reported in the local Asian community despite there being many cases in the same area in the non-Asian community [59]. Although it was hypothesized that cultural factors partially explained this observation, serosurvey data provided evidence for an underlying immunity as many members of the Asian community had originated from areas of Pakistan with high rates of Coxiella burnetii infection in sheep [59]. Factors other than environmental exposure can also affect susceptibility, e.g. socioeconomic status, which is an important co-factor for the prevalence of $H$. pylori in the US adult population [60]. The available evidence for the occurrence of subclinical infections demonstrates that they can be multifactorial and may be related to the type or level of exposure, the presence of potential immunity in the host, or agent-specific factors, such as genotypic variation in virulence. Whatever the mechanism, the presence of subclinical zoonotic infections in human populations, together with associated asymptomatic carriage, gives rise to a number of important issues for the epidemiology and control of these diseases.

\section{Community epidemiology and the public health significance of subclinical zoonotic infections in humans}

The emergence of several zoonotic diseases of major public health significance, e.g. swine influenza A(H1N1), E. coli O157, vCJD, and SARS, have highlighted the importance of early surveillance. Essential to mitigating the spread of communicable diseases is the implementation of control measures, which includes horizon-scanning and risk assessment. However, the presence of subclinical cases can present a barrier to effective disease control as infections often escape detection. Subsequently, under-ascertainment of cases may underestimate the extent of an outbreak, and facilitate continued transmission, or lead to bias in an epidemiological investigation, e.g. by the misclassification of controls in case-control studies. Further, the presence of subclinical or mild infection in a proportion of hosts may provide an evolutionary advantage to an emerging pathogen, facilitating onward transmission within animal and/or human populations. Asymptomatic carriers can still excrete infective organisms, and may pass the infection on to others, although for gastrointestinal infections, transmission is more likely from clinical cases, especially where cases exhibit diarrhoea (Fig. 1).

Person-to-person spread of zoonoses is most usually considered in the control of gastrointestinal infections such as Salmonella and E. coli O157. Horizontal transmission of $E$. coli $\mathrm{O} 157$ spread via the faecaloral route can occur in families and in the wider community. However, secondary transmission of salmonellosis during outbreaks may vary depending on the outbreak setting and the strain of pathogen, and is often dependent on the population at risk. In a large outbreak on the Navajo Nation Indian 


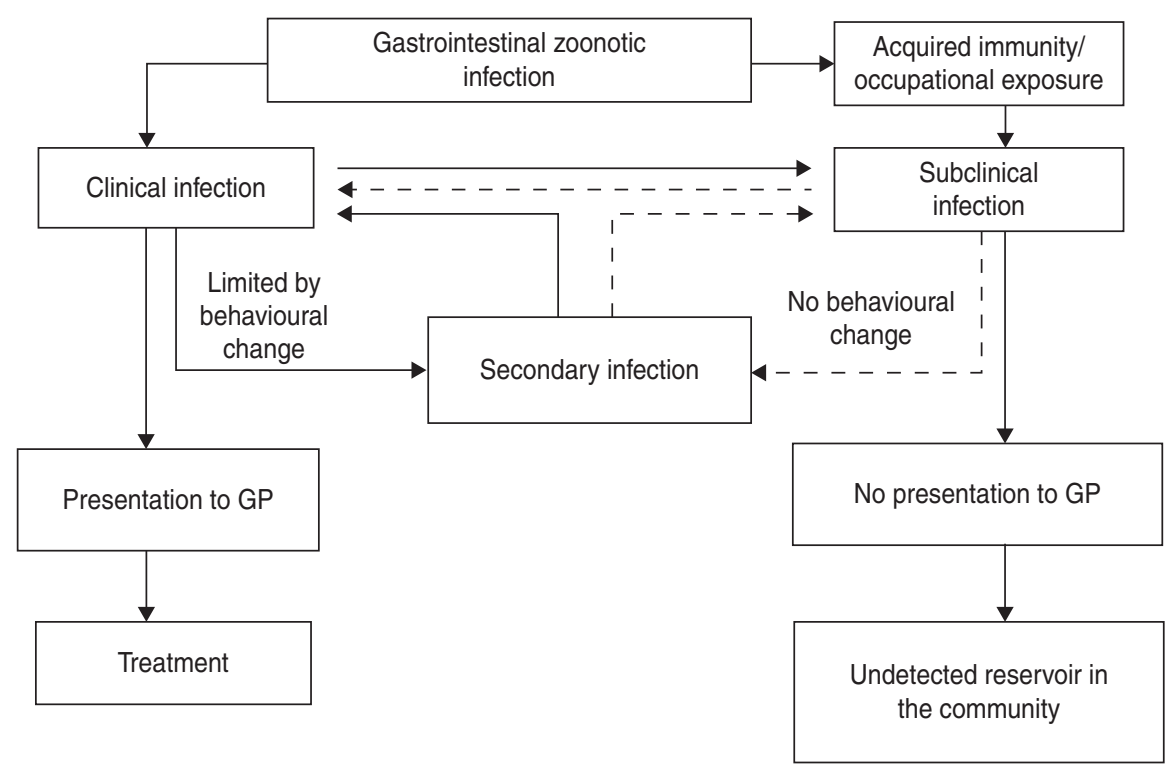

Fig. 1. Infection pathways and potential for the maintenance of asymptomatic carriage of gastrointestinal zoonotic pathogens.

Reserve in the USA, which involved 3400 cases, no secondary infections were reported [61], whereas in hospital outbreaks in the UK during the 1980s person-to-person transmission was a significant factor [62]. Secondary transmission of E. coli $\mathrm{O} 157$ is relatively common [63], and of 157 cases identified in a school outbreak in Wales, UK, 48 cases were attributed to secondary transmission [64]. By contrast, person-to-person spread of Campylobacter is rare and family clusters are seldom seen [65], and although secondary transmission following point-source outbreaks does occur, it is uncommon [66]. In a recent outbreak of $E$. coli $\mathrm{O} 157$ on a petting farm in the UK, of 93 cases of illness, 65 were attributed to primary infection, 13 to secondary infection and 15 were asymptomatic cases [67]. While it is theoretically possible that some of the secondary cases of infection could be linked to direct person-to-person spread from some of the asymptomatic cases, quantifying this risk during an outbreak scenario would prove very difficult. However, the potential for asymptomatic human carriage as an epidemiological variable should not be discounted [68].

Guidelines exist in the UK for the prevention of person-to-person spread of gastrointestinal infections in general [69], and E. coli O157 in particular [70]. These guidelines consider the public health management of cases and contacts and the control of secondary transmission. Special attention is given to hygiene precautions, such as hand washing, decontamination, exclusion from work, school and other institutional settings, and identifying population groups at greater risk of spreading infection, e.g. children aged $<5$ years attending pre-school facilities, people working with ready-to-eat food and health and social care staff who have direct contact with individuals, for whom a gastrointestinal infection could have serious consequences. Outbreaks in children's nurseries in which an explicit link between human asymptomatic carriers of E. coli $\mathrm{O} 157$ and person-to-person infection have been reported [71]. Given the frequency of secondary transmission by E. coli O157, and the potential severity of outcomes of infection, the prompt separation of primary cases of E. coli $\mathrm{O} 157$ from young household contacts has been recommended [72].

The theoretical possibility of person-to-person infection from asymptomatic carriers poses a significant demand on the monitoring and control of disease outbreaks. Methicillin-resistant Staphylococcus aureus (MRSA) is an example of a disease with evidence of asymptomatic person-to-person infection, e.g. healthcare workers [73], and recent reports also suggest a zoonotic pathway through professional contact with animals [74-76]. Asymptomatically colonized individuals can transmit MRSA to others [77], and in so doing can unknowingly act as reservoirs for the disease [73], particularly in nosocomial situations [78-81]. Although the infection pathways for zoonotic MRSA transmission are different to E. coli O157, 
Salmonella, Campylobacter and Cryptosporidium, the role of occupational exposure, asymptomatic carriage and person-to-person spread of MRSA gives rise to a number of important implications relating to the control and surveillance of gastrointestinal zoonotic diseases.

Livestock farmers and veterinarians in this context are an occupational group analogous to healthcare workers and should be considered at risk of becoming asymptomatic carriers due to their regular handling of potentially infected animals. Therefore, protective measures for this group are important [82]; although approaches proposed for healthcare workers in hospital settings, e.g. regular screening and decolonization of carriers [83], would be impractical for workers in the livestock sector. Screening farmers for occupationally acquired diseases would be logistically problematical owing to the number of farms, their type and the practicality of regularly screening in geographically isolated locations. Veterinarians pose a potentially greater threat for the dispersal of these types of pathogen than farmers due to their multiple daily farm visits, and subsequently have greater potential for asymptomatic person-to-person contact within the more densely populated urban and semi-urban communities.

The importance of asymptomatic carriage in causing secondary infection was highlighted during a retrospective cohort study following an outbreak of E. coli $\mathrm{O} 157$ in the UK [72]. Although it would be fairly straightforward to set up a similar study to quantify the spread of zoonoses from primary symptomatic cases to asymptomatic secondary cases, future epidemiological investigations need to focus on quantifying the spread from asymptomatic carriers. However, identifying clinical cases that result from the secondary transmission from asymptomatic primary cases would be chronologically difficult during an outbreak setting, as the investigation usually begins with the symptomatic case.

\section{Potential mitigation strategies}

If asymptomatic carriers are a source of infection then there may be an economic case for identifying and treating asymptomatic carriage if the costs of screening and treatment are less than the costs of identifying and treating those individuals infected by asymptomatic hosts. One strategy to encourage high-risk occupational groups to seek tests and treatment would be to impose penalties and/or positive incentives.
The latter could involve payment for regular screening for high-risk asymptomatic carriage groups such as veterinarians. However, surveillance of veterinarians and farmers may prove to be controversial and a need to adhere to strict confidentiality guidelines essential [84]. As a control measure the weekly voluntary screening of veterinarians appears to be technically feasible and coupled with an overall improvement in hygiene practices may provide sufficient protection from transmitting both clinical and subclinical infections $[42,84]$. Schemes which aim to reduce the risk posed by asymptomatic carriers in the food chain are mostly not predicated on the assumption that there is some intervention that will remove gastrointestinal zoonotic pathogens from asymptomatic hosts. Rather, those hosts are removed from food handling until such time as the organism can no longer be recovered from their stool. Such interventions are not available for all zoonoses, and even if they were, they would need to be taken regularly in order to counter host re-colonization. The imposition of any programme aimed at reducing the risk from asymptomatic carriers may be best focused on groups with a higher risk of transmitting zoonoses to others and lower probabilities of infection, e.g. farmers and veterinarians.

In theory, a prospective cohort study could be set up following a cohort of high-risk individuals (high risk of acquiring a zoonotic infection with a low risk of clinical symptoms, but a high risk of transmitting it on) and collecting detailed information about their contacts. In practice, this would probably need a large number of people, as risk of secondary spread would be low, and would therefore not be practical. Another approach would be to simply screen a healthy occupational cohort for markers of infection, screen the contacts of both positives and negatives and simply examine statistically whether contacts of positives are more likely to be positive. However, this would likely be confounded by the fact that contacts would have other common sources of infection, rather than linearly transmitting to each other.

The effective management of such diseases is contingent upon substantive empirical information underpinning policy decisions. The nature of emerging diseases often requires policy makers and scientists to make choices about disease control in the absence of hard empirical data. Although when the efficacy of a large number of intervention strategies requires such thorough evaluation the task can be cognitively taxing. To overcome such obstacles, recent research 
has employed a range of innovative techniques including the market research tool 'Best-Worst Scaling' designed to facilitate the elicitation of opinion from both health experts and land managers. The common objective of such an approach is to identify interventions considered both effective in the control of pathogen movement within the wider environment and practical to implement [85]. The compliance of key stakeholders such as farmers and veterinarians, however, is critical to successful policy implementation.

This review has highlighted a number of important gaps in our knowledge about the significance of asymptomatic carriage and subclinical infection, and exposed a lack of understanding about the risks posed by subclinical infection in different parts of the food chain from field to fork. This includes the rate of asymptomatic carriage in different occupational groups (farmers, veterinarians, abattoir and processing workers, food manufacturers and handlers) and the risk of person-to-person transmission from asymptomatic carriers. It is therefore essential that future research is concentrated towards understanding the role occupational exposure and asymptomatic carriage play in outbreak scenarios and the epidemiological significance of subclinical infection in the community.

\section{ACKNOWLEDGEMENTS}

This research was undertaken as part of a project funded under the UK Research Councils' Rural Economy and Land Use (Relu) programme entitled 'Reducing E. coli O157 Risk in Rural Communities' (RES-229-31-0003). Relu is funded jointly by the Economic and Social Research Council, the Biotechnology and Biological Sciences Research Council and the Natural Environment Research Council, with additional funding from the Department for Environment, Food and Rural Affairs and the Scottish Government.

\section{DECLARATION OF INTEREST}

None.

\section{REFERENCES}

1. Adak GK, Long SM, O'Brien SJ. Trends in indigenous foodborne disease and deaths, England and Wales: 1992 to 2000. Gut 2002; 51: 832-841.
2. Mead PS, et al. Food-related illness and death in the United States. Emerging Infectious Diseasess 1999; 5: 607-625.

3. Centers for Disease Control. Q fever among slaughterhouse workers - California. Morbidity and Mortality Weekly Report 1986; 35: 223-2264.

4. Thomas DR, et al. Zoonotic illness - determining risks and measuring effects - association between current animal exposure and a history of illness in a well characterised rural population in the UK. Journal of Epidemiology and Community Health 1994; 48: 151155.

5. Whitney EAS, et al. Seroepidemiologic and cccupational risk survey for Coxiella burnetii antibodies among US veterinarians. Clinical Infectious Diseases 2009; 48: 550-557.

6. Silvestro L, et al. Asymptomatic carriage of Verocytotoxin-producing Escherichia coli $\mathrm{O} 157$ in farm workers in Northern Italy. Epidemiology \& Infection 2004; 132: 915-919.

7. Gladstone BP, et al. Polymerase chain reaction in the detection of an 'outbreak' of asymptomatic viral infections in a community birth cohort in south India. Epidemiology \& Infection 2008; 136: 399-405.

8. Wilson JB, et al. Vero cytotoxigenic Escherichia coli infection in dairy farm families. Infectious Disease 1996; 174: 1021-1027.

9. Evans J, et al. Evidence of persisting serum antibodies to Escherichia coli $\mathrm{O} 157$ lipopolysaccharide and Verocytotoxin in members of rural communities in England. European Journal of Epidemiology 2000; 16: 885-889.

10. Reymond D, et al. Neutralizing antibodies to Escherichia coli Vero cytotoxin 1 and antibodies to O157 lipopolysaccharide in healthy farm family members and urban residents. Journal of Clinical Microbiology 1996; 34: 2053-2057.

11. Belongia EA, et al. Diarrhea incidence and farm-related risk factors for Escherichia coli O157:H7 and Campylobacter jejuni antibodies among rural children. Journal of Infectious Diseases 2003; 187: 1460-1468.

12. Park J, et al. Prevalence of Shiga toxin-encoding genes and risk factors among dairy farmers in Gyeonggi Province, Korea. Scandinavian Journal of Infectious Diseases 2012; 43: 275-279.

13. Quilliam RS, et al. Seroprevalence and risk factors associated with Escherichia coli $\mathrm{O} 157$ in a farming population. Zoonoses and Public Health 2012; 59: 83-88.

14. Jones DM, Robinson DA. Occupational exposure to Campylobacter jejuni infection. Lancet 1981; 1: 440-441.

15. Price LB, et al. Neurologic symptoms and neuropathologic antibodies in poultry workers exposed to Campylobacter jejuni. Journal of Occupational \& Environmental Medicine 2007; 49: 748-755.

16. Ellis A, et al. Outbreak of Campylobacter infection among farm workers: an occupational hazard. Canadian Communicable Disease Report 1995; 21: 153156. 
17. Wilson IG. Airborne Campylobacter infection in a poultry worker: case report and review of the literature. Communicable Disease \& Public Health 2004; 7: 349-353.

18. Cawthraw SA, et al. Antibodies, directed towards Campylobacter jejuni antigens, in sera from poultry abattoir workers. Clinical \& Experimental Immunology 2000; 122: 55-60.

19. Kapperud G, et al. Factors associated with increased and decreased risk of Campylobacter infection: a prospective case-control study in Norway. American Journal of Epidemiology 2003; 158: 234-242.

20. Studahl A, Andersson Y. Risk factors for indigenous Campylobacter infection: a Swedish case-control study. Epidemiology \& Infection 2000; 125: 269-275.

21. Eberhart-Phillips J, et al. Campylobacteriosis in New Zealand: results of a case-control study. Journal of Epidemiology \& Community Health 1997; 51: 686-691.

22. Adak GK, et al. The public health laboratory service national case-control study of primary indigenous sporadic cases of Campylobacter infection. Epidemiology \& Infection 1995; 115: 15-22.

23. Robinson DA, Jones DM. Milk-borne Campylobacter infection. British Medical Journal 1981; 282: 1374-1376.

24. Blaser MJ, Sazie E, Williams LP. The influence of immunity on raw milk-associated Campylobacter infection. Journal of the American Medical Association 1987; 257: 43-46.

25. Goh S, et al. Sporadic cryptosporidiosis, North Cumbria, England, 1996-2000. Emerging Infectious Diseases 2004; 10: 1007-1015.

26. Hunter PR, et al. Sporadic cryptosporidiosis casecontrol study with genotyping. Emerging Infectious Diseases 2004; 10: 1241-1249.

27. Pollock KGJ, et al. Spatial and temporal epidemiology of sporadic human cryptosporidiosis in Scotland. Zoonoses and Public Health 2009; 57: 487-492.

28. Chalmers RM, et al. Epidemiology of anthroponotic and zoonotic humancryptosporidiosis in England and Wales, 2004-2006. Epidemiology \& Infection 2011; 139: 700-712.

29. Casemore DP. The antibody response to cryptosporidium: development of a serological test and its use in a study of immunologically normal persons. Journal of Infection 1987; 14: 125-134.

30. Garcia-Rodriguez JA, et al. The seroepidemiology of Cryptosporidium species in different population groups in Spain. Serodiagnosis \& Immunotherapy in Infectious Disease 1989; 3: 367-373.

31. Lengerich EJ, et al. Increased exposure to Cryptosporidia among dairy farmers in Wisconsin. Journal of Infectious Diseases 1993; 167: 1252-1255.

32. Gait R, et al. Outbreak of cryptosporidiosis among veterinary students. Veterinary Record 2008; 162: 843845.

33. Chalmers RM, Davies A. Minireview: clinical cryptosporidiosis. Experimental Parasitology 2010; 124: 138-146.

34. Swift L, Hunter PR. What do negative associations between potential risk factors and illness in analytical epidemiological studies of infectious disease really mean? European Journal of Epidemiology 2004; 19: 219-223.

35. Frost FJ, et al. How clean must our drinking water be: the importance of protective immunity. Journal of Infectious Diseases 2005; 191: 809-814.

36. Okhuysen PC, et al. Susceptibility and serologic response of healthy adults to reinfection with Cryptosporidium parvum. Infection and Immunity 1998; 66: 441-443.

37. Chappell CL, et al. Infectivity of Cryptosporidium parvum in healthy adults with pre-existing anti-C. parvum serum immunoglobulin G. American Journal of Tropical Medicine \& Hygiene 1999; 60: 157-164.

38. Fone DL, Barker RM. Associations between human and animal infections with Salmonella Typhimurium DT104 in Herefordshire. Communicable Disease Reports. CDR Review 1994; 4: R136-R140.

39. Baker MG, et al. A recurring salmonellosis epidemic in New Zealand linked to contact with sheep. Epidemiology \& Infection 2007; 135: 76-83.

40. Cherry B, et al. Salmonella Typhimurium outbreak associated with veterinary clinic. Emerging Infectious Diseases 2004; 10: 2249-2251.

41. Wright JG, et al. Multidrug-resistant Salmonella Typhimurium in four animal facilities. Emerging Infectious Diseases 2005; 11: 1235-1241.

42. Wright JG, et al. Infection control practices and zoonotic disease risks among veterinarians in the United States. Journal of the American Medical Association 2008; 232: 1863-1872.

43. Shlim DR, et al. Persistent high risk of diarrhea among foreigners in Nepal during the first 2 years of residence. Clinical Infectious Diseases 1999; 29: 613-616.

44. Wenneras C, Erling VA. Prevalence of enterotoxigenic Escherichia coli-associated diarrhoea and carrier state in the developing world. Journal of Health \& Population Nutrition 2004; 22: 370-382.

45. Havelaar AH, et al. Immunity to Campylobacter: its role in risk assessment and epidemiology. Critical Reviews in Microbiology 2009; 35: 1-22.

46. Hoge CW, et al. Epidemiology of diarrhea among expatriate residents living in a highly endemic environment. Journal of the American Medical Association 1996; 275: 533-538.

47. Hong $\mathbf{S}$, et al. Anti-Shiga toxin immunoglobulin $\mathrm{G}$ antibodies in healthy South Korean slaughterhouse workers. Scandinavian Journal of Infectious Diseases 2012; 44: 168-173.

48. Hellard ME, et al. Prevalence of enteric pathogens among community based asymptomatic individuals. Journal of Gastroenterology \& Hepatology 2000; 15: 290-293.

49. Haffejee IE, Windsor I, Moosa A. Asymptomatic rotavirus infections among normal Indian children in Chatsworth, Durban. South African Medical Journal 1989; 76: 599-601.

50. Morrow AL, et al. Human milk oligosaccharides are associated with protection against diarrhea in breast-fed infants. Journal of Pediatrics 2004; 145: 297-303. 
51. Tikare NV, Mantur BG, Bidari LH. Brucellar meningitis in an infant - evidence for human breast milk transmission. Journal of Tropical Pediatrics 2008; 54: 272-274.

52. Celebi G, et al. Asymptomatic Brucella bacteraemia and isolation of Brucella melitensis biovar 3 from human breast milk. Scandinavian Journal of Infectious Diseases 2007; 39: 205-208.

53. Clemens J, et al. Sociodemographic, hygienic and nutritional correlates of Helicobacter pylori infection of young Bangladeshi children. Pediatric Infectious Disease Journal 1996; 15: 1113-1118.

54. Alikhani MY, et al. Prevalence of enteropathogenic and Shiga toxin-producing Escherichia coli among children with and without diarrhoea in Iran. Journal of Health \& Population Nutrition 2007; 25: 88-93.

55. Lindesmith $\mathbf{L}$, et al. Human susceptibility and resistance to Norwalk virus infection. Nature Medicine 2003; 9: 548-553.

56. Burgner D, Jamieson SE, Blackwell JM. Genetic susceptibility to infectious diseases: big is beautiful, but will bigger be even better? Lancet Infectious Diseases 2006; 6: 653-663.

57. Ottenhoff T, et al. Genetics, cytokines and human infectious disease: lessons from weakly pathogenic mycobacteria and salmonellae. Nature Genetics 2002; 32: 97-105.

58. Janssen R, et al. Host-pathogen interactions in Campylobacter infections: the host perspective. Clinical Microbiology Review 2008; 21: 505-518.

59. Hawker JI, et al. A large outbreak of Q fever in the West Midlands: windborne spread into a metropolitan area? Communicable Disease \& Public Health 1998; 1: 180-187.

60. Everhart JE, et al. Seroprevalence and ethnic differences in Helicobacter pylori infection among adults in the United States. Journal of Infectious Diseases 2000; 181: 1359-1363.

61. Horowitz MA, et al. Large outbreak of foodborne Salmonellosis on Navajo Nation Indian Reservation, epidemiology and secondary transmission. American Journal of Public Health 1977; 67: 1071-1076.

62. Palmer SR, Rowe B. Comminicable diseases - investigation of outbreaks of Salmonella in hospitals. British Medical Journal 1983; 287: 891-893.

63. Parry SM, Salmon RL. Sporadic STEC O157 infection: secondary household transmission in Wales. Emerging Infectious Diseases 1998; 4: 657-661.

64. Pennington H. The Public Inquiry into the September 2005 Outbreak of E. coli O157 in South Wales. London: The Stationery Office, HMSO, 2009.

65. Cowden J. Campylobacter - epidemiologic paradoxes. British Medical Journal 1992; 305: 132-133.

66. Evans MR, et al. A milk-borne Campylobacter outbreak following an educational farm visit. Epidemiology \& Infection 1996; 117: 457-462.

67. Defra. Zoonoses Report 2009 UK. Department for the Environment, Food and Rural Affairs, London, 2009.

68. David ST, et al. Petting zoo-associated Escherichia coli $\mathrm{O} 157: \mathrm{H} 7$ - secondary transmission, asymptomatic infection, and prolonged shedding in the classroom.
Canadian Communicable Disease Report 2004; 30: 173-80.

69. Working Group of the former PHLS Advisory Committee on Gastrointestinal Infections. Preventing person-to-person spread following gastrointestinal infections: guidelines for public health physicians and environmental health officers. Communicable Disease \& Public Health 2004; 7: 362-384.

70. Subcommittee of the PHLS Advisory Committee on Gastrointestinal Infections. Guidelines for the control of infection with verocytotoxin producing Eschericia coli (VTEC). Communicable Disease \& Public Health 2000; 3: 14-23.

71. Al-Jader L, et al. Outbreak of Escherichia coli $\mathrm{O} 157$ in a nursery: lessons for prevention. Archives of Disease in Childhood 1999; 81: 60-63.

72. Werber D, et al. Preventing household transmission of Shiga toxin producing Escherichia coli O157 infection: Promptly separating siblings might be the key. Clinical \& Infectious Diseases 2008; 46: 1189-1196.

73. Albrich WC, Harbarth S. Health-care workers: source, vector, or victim of MRSA? Lancet Infectious Diseases 2008; 8: 289-301.

74. Voss A, et al. Methicillin-resistant Staphylococcus aureus in pig farming. Emerging Infectious Diseases 2005; 11: $1965-1966$.

75. Wulf MWH, et al. Prevalence of methicillin-resistant Staphylococcus aureus among veterinarians: an international study. Clinical Microbiology \& Infection 2008; 14: 29-34.

76. Huber H, et al. Prevalence and characteristics of methicillin-resistant Staphylococcus aureus in humans in contact with farm animals, in livestock, and in food of animal origin, Switzerland, 2009. Eurosurveillance 2010; 15: 19542

77. Meier PA, et al. A prolonged outbreak of methicillin resistant Staphylococcus aureus in the burn unit of a tertiary medical center. Infection Control \& Hospital Epidemiology 1996; 17: 798-802.

78. Blok H, et al. Role of healthcare workers in outbreaks of methicillin-resistant Staphylococcus aureus: a 10year evaluation from a Dutch university hospital. Infection Control \& Hospital Epidemiology 2003; 24: 679-685.

79. Saiman L, et al. An outbreak of methicillin-resistant Staphylococcus aureus in a neonatal intensive care unit. Infection Control \& Hospital Epidemiology 2003; 24: 317-321.

80. Bertin ML, et al. Outbreak of methicillin-mesistant Staphylococcus aureus colonization and infection in a neonatal intensive care unit epidemiologically linked to a healthcare worker with chronic otitis. Infection Control \& Hospital Epidemiology 2006; 27: 581-585.

81. Grundmann H, et al. Emergence and resurgence of meticillin-resistant Staphylococcus aureus as a publichealth threat. Lancet 2006; 368: 874-885.

82. Garcia-Graells C, et al. Livestock veterinarians at high risk of acquiring methicillin-resistant Staphylococcus aureus ST398. Epidemiology \& Infection 2012; 140: 383-389. 
83. Cookson B, et al. Meticillin-resistant Staphylococcus aureus (MRSA): screening and decolonisation. International Journal of Antimicrobial Agents 2011; 37: 195-201.

84. Catry B, et al. Reflection paper on MRSA in food-producing and companion animals: epidemiology and control options for human and animal health. Epidemiology \& Infection 2010; 138: 626 644.

85. Cross P, Rigby D, Edwards-Jones G. Eliciting expert opinion on the effectiveness and practicality of interventions in the farm and rural environment to reduce human exposure to Escherichia coli O157. Epidemiology \& Infection 2012; 40: 643-654. 